This is the peer reviewed version of the following article: Q. Wang, J. Frisch, M. Herder, S. Hecht, N. Koch, ChemPhysChem 2017, 18,722-727, which has been published in final form at http://onlinelibrary.wiley.com/doi/10.1002/cphc.201601442/full. This article may be used for non-commercial purposes in accordance with Wiley Terms and Conditions for Self-Archiving. Supporting Information is available free of charge following the previous link.

\title{
Electronic Properties of Optically Switchable Photochromic Diarylethene Molecules at the Interface with Organic Semiconductors
}

\author{
Qiankun Wang ${ }^{1}$, Johannes Frisch ${ }^{1}$, Martin Herder ${ }^{2}$, Stefan Hecht $^{2}$, Norbert Koch ${ }^{1 *}$ \\ ${ }^{1}$ Institut für Physik \& IRIS Adlershof, Humboldt-Universität zu Berlin, Brook-Taylor- \\ Straße 6, 12489 Berlin, Germany \\ ${ }^{2}$ Department of Chemistry \& IRIS Adlershof, Humboldt-Universität zu Berlin, Brook- \\ Taylor-Straße 2, 12489 Berlin, Germany \\ *e-mail: norbert.koch@physik.hu-berlin.de
}

\begin{abstract}
Light-switching induced changes in the electronic properties of photochromic diarylethene, i.e., 1,2-bis(2-methyl-5-p-tolylthiophen-3-yl)cyclopent-1-ene (DAE1), thin films at interfaces to a gold electrode and two polymer semiconductors were investigated by direct and inverse photoelectron spectroscopy. The photoisomerization was achieved by in situ irradiation of ultraviolet and visible light. Efficient and reversible switching between the open and closed isomers of DAE1 was evidenced at all interfaces, with profound impact on the energy level alignment. The frontier occupied level of DAE1 changes by $0.8 \mathrm{eV}$ with respect to the Au Fermi level upon switching. Corresponding sizable changes in the electron and transport level offsets between the two polymers and DAE1 in its open and closed form were determined. This gives rise to fundamentally
\end{abstract}


different functionality of these interfaces in terms of charge transport. Our study proves the viability of light controlled energy level manipulation at various interfaces in photoswitchable opto-electronic devices.

Keywords: photochromic diarylethenes, photoisomerization, energy level alignment, charge transport.

\section{Introduction}

Photochromic organic molecules (PCOMs), such as stilbenes, azobenzenes, diarylethenes (DAEs), and spiropyrans have attracted increasing interest for the development of low-cost, easy-processing multifunctional devices, including phototransistors ${ }^{[1]}$ and optical memories. ${ }^{[2,3]}$ Due to the photochromism, these molecules can switch reversibly between two isomers by light irradiation with different wavelengths. ${ }^{[4-7]}$ Switching between the two isomers fundamentally changes the electronic properties of the molecules, e.g., the optical gap, molecular dipole moment, molecular electron affinity, and ionization energy. ${ }^{[8-12]}$. Using PCOMs in blends with organic semiconductors or as photo-addressable interlayer between an organic semiconductor and an electrode, the photo-induced switching can tune charge transport as well as charge injection (extraction) properties in photoswitchable devices. ${ }^{[13-16]}$ The underlying reason is that the energy level offset between the highest occupied molecular orbital (HOMO) level of PCOMs and either the Fermi level $\left(E_{\mathrm{F}}\right)$ of the electrodes and/or the valence band of the organic semiconductor in the device are minimized for one isomer state and maximized for the other, as selected by appropriate photo-induced 
switching. Analogous arguments apply when considering the lowest unoccupied molecular orbital (LUMO) level and the conduction band. Consequently, the electrical properties of the devices are modulated by either the formation of photoswitchable trap states or energy barriers for charges at organic/PCOM or metal/PCOM interfaces, and multifunctionality is realized as the device can be addressed electrically and optically. ${ }^{[13}$, ${ }^{15]}$ The application of PCOMs in electronic devices requires high thermal stability of the material in its individual isomer state, and at the same time, a highly efficient and robust photoisomerization process. Therefore, DAE molecules show promising potential due to their high fatigue resistance and thermal bistability. ${ }^{[6,17]}$ Ultraviolet photoemission spectroscopy (UPS) studies on thin films of DAE derivatives revealed a large difference of the ionization energy (IE) between the open and closed isomers of approximately $1 \mathrm{eV} \cdot{ }^{[10]}$ It was reported that this change in IE can be used to alter the current through the channel of organic field effect transistors (OFET) where DAE molecules were blended into the polymeric semiconductor poly(hexylthiophene) (P3HT) ${ }^{[12,13]}$ Nevertheless, the energy level alignment (ELA) at the electrode/DAE as well as the organic semiconductor/DAE interfaces is not comprehensively understood. Particularly with respect to metal electrodes, the energy position of DAE frontier levels is yet unknown, and even the ability to photo-switch the molecules in proximity to the metal can questioned due to dipole selection rules. In the context of energy level alignment, it has been shown that the Schottky-Mott limit (vacuum level alignment) often fails to predict the energy level difference, e.g., between the highest occupied $\left(\Delta_{\mathrm{h}}\right)$ and lowest unoccupied molecular orbital $\left(\Delta_{\mathrm{e}}\right)$ with respect to the Fermi level of the system at metal/organic and organic/organic interfaces, as shown in Fig. 1a. Therefore, a detailed 
analysis of the electronic properties and interface energetics of DAE-based (bulk and planar) heterojunctions is of importance for understanding the impact on charge injection and transport, and for optimizing photoswitchable devices.

Here, the frontier electronic structure of interfaces with a photochromic diarylethene derivative, 1,2-bis(2-methyl-5-p-tolylthiophen-3-yl)cyclopent-1-ene (DAE1) was investigated via photoemission spectroscopy (PES) measurements, with an emphasis on the impact of in situ PCOM light-induced switching. The absorption spectra and chemical structure of DAE1 are shown in Fig. 1b. Switching between the open and the closed forms (DAE1-o and DAE1-c, respectively) was followed in situ upon appropriate illumination with ultraviolet $(\lambda=365 \mathrm{~nm})$ and visible light $(\lambda>450 \mathrm{~nm})$, respectively. As the charge transport gap of DAE1 films was determined by the combination of UPS and inverse photoemission spectroscopy (IPES), we reveal the full electronic structure of DAE1 on a gold electrode, and demonstrate photo-induced switching. The ELA at the interface between DAE1 and a prototypical hole conducting polymer poly(3hexylthiophene) (P3HT) and an electron conducting polymer poly $\{[\mathrm{N}, \mathrm{N} 0-\mathrm{bis}(2-$ octyldodecyl)naphthalene-1,4,5,8-bis(dicarboximide)-2,6-diyl]-alt-5,50-(2,20-

bithiophene)\} (N2200) (both chemical structures shown in Fig. 1c) were determined, again including in situ switching. We find that the photoisomerization process has a huge influence on interface energy level offsets, leading to photo-tunable hole (electron) transport. This provides a reliable rationale for the photo-control of charge transport in organic electronic devices that employ PCOMs.

\section{Experimental section}


Indium-tin-oxide (ITO) coated glass (sheet resistance 15-30 $\Omega$ ) and Au covered $\mathrm{Si}$ wafers (with $3 \mathrm{~nm}$ Ti as adhesion layer) were used as substrates; they were cleaned by sonication in acetone and isopropanol before spin coating organic layers. All subsequent sample preparation steps were performed in the inert atmosphere $\left(\mathrm{N}_{2}\right)$ of a glove-box. DAE1 molecules (details of the synthesis are described in Ref. 13) dissolved in chloroform solvent were directly spin coated onto Au substrates. The thickness of the films was varied by adjusting spinning speed and solution concentration. Consequently, $2 \mathrm{~nm}$ and $10 \mathrm{~nm}$ DAE1 layers were obtained by spin coating $5 \mathrm{mg} / \mathrm{mL}$ and $1 \mathrm{mg} / \mathrm{mL}$ solution at the speed of $25 \mathrm{rps}$ and $13 \mathrm{rps}$, respectively.

For interfaces with polymeric semiconductors, the polymers P3HT and N2200, dissolved in chloroform, were deposited on PEDOT:PSS pre-covered ITO/glass by spin coating with a speed of $25 \mathrm{rps}$. Samples were subsequently annealed at $200{ }^{\circ} \mathrm{C}(\mathrm{P} 3 \mathrm{HT})$ and $120^{\circ} \mathrm{C}(\mathrm{N} 2200)$ on a hot plate for $30 \mathrm{~min}$. After annealing, samples were subsequently washed via spin coating pure chloroform solvent to obtain ultrathin polymer films. ${ }^{[27,28]}$ Afterwards, DAE1 molecules were sequentially deposited with increasing concentrations and different spin speed, i.e., firstly, $0.5 \mathrm{mg} / \mathrm{mL}(15 \mathrm{rps})$ to obtain a film thickness of ca. $1 \mathrm{~nm}$, secondly, $2 \mathrm{mg} / \mathrm{mL}$ (17 rps), and thirdly, $4 \mathrm{mg} / \mathrm{mL}$ (20 rps), the total nominal thickness is ca. $5 \mathrm{~nm}$, which was determined by atomic force microscopy (AFM) measurements. After every deposition step, samples were transferred from the glove-box to the ultrahigh vacuum (UHV) chamber for UPS/XPS measurements without exposure to ambient atmosphere.

UPS experiments were performed at a custom-made multi-chamber UHV system, which consists of a fast load lock (base pressure $<1 \times 10^{-7}$ mbar), a sample preparation 
chamber $\left(<5 \times 10^{-9} \mathrm{mbar}\right)$, and an analysis chamber $\left(<2 \times 10^{-10} \mathrm{mbar}\right)$. Photoelectrons were excited with Al-foil attenuated $\mathrm{He} \mathrm{I}(h v=21.22 \mathrm{eV})$ radiation at very low excitation density (approx. 20 times attenuated compared to the standard sources); under these conditions, radiation damage and charging of the samples was negligible (for more details, see Fig. S1 in the Supporting Information). Photoelectrons were collected and measured with a hemispherical spectrometer (SPECS Phoibos 100); the total instrumental energy resolution was $150 \mathrm{meV}$. The secondary electron cutoff (SECO) spectra were recorded with samples biased at $-10 \mathrm{~V}$ to clear the analyzer work function. X-ray photoemission spectroscopy (XPS) measurements using $\mathrm{Al} \mathrm{K \alpha}$ source $(1486.6 \mathrm{eV})$ were conducted to study the core levels. The binding energy scale was calibrated by determining the Au4f $f_{7 / 2}$ peak position (XPS) and Fermi level edge of the Au film (UPS), which was cleaned by $\mathrm{Ar}^{+}$ion sputtering. The Fermi level is referred to as the zero binding energy in all the UPS and XPS spectra. IPES was performed in the same system with a custom setup (incident electron energy range: $5-15 \mathrm{eV}, \mathrm{NaCl}$ coated photocathode, SrF-window). During sample preparation, transfer, and measurements, the samples were either kept in dark or exposed to low-intensity red light to avoid unintended switching of DAE1. For switching, the samples were irradiated in situ during UPS measurements through a viewport on the UHV chamber with visible or ultraviolet (UV) light. Visible light $(\lambda>450 \mathrm{~nm})$ was obtained by putting a $450 \mathrm{~nm}$ cut-off filter in front of a white light lamp (SoLux, $4700 \mathrm{~K}, 35 \mathrm{~W}, 36^{\circ}$, EIKO); the measured photon intensity $\left(\mathrm{I}_{0}\right)$ was at ca. $200 \mathrm{~mW} / \mathrm{cm}^{2}$. UV light ( $\lambda$ centered at $365 \mathrm{~nm}$ ) was provided by a high power UV-LED (Thorlabs, ML365) mounted with an adjustable collimator; the measured $\mathrm{I}_{0}$ was ca. $100 \mathrm{~mW} / \mathrm{cm}^{2}$. All measurements were performed at room temperature. 



\section{Results and Discussion}

To probe the photoisomerization of DAE1 switches and their ELA at the Au/DAE1 interface, at first a DAE1-c film with a thickness of approximately $10 \mathrm{~nm}$ on a polycrystalline Au substrate was studied by UPS. During the measurements, visible and UV light were employed to achieve photoswitching. As shown in Fig. 2a, the measured onset of the HOMO level and work function (WF) for the DAE1-c film were $0.56 \mathrm{eV}$ and $4.45 \mathrm{eV}$, respectively. Its IE value thus was $5.01 \mathrm{eV}$, in line with the reported values. ${ }^{[10]}$ The subsequent, light-induced photochromic reaction is accompanied by both changes of the film color (from pink to transparent) and of valence band region spectra. The HOMO feature of the DAE1-c film [centered at $\sim 1 \mathrm{eV}$ binding energy (BE)] completely disappeared after $60 \mathrm{~s}$ of visible light illumination, and instead a new occupied feature with the onset at $\sim 1.32 \mathrm{eV} \mathrm{BE} \mathrm{emerged,} \mathrm{indicating} \mathrm{the} \mathrm{formation} \mathrm{of} \mathrm{DAE1-o} \mathrm{isomers.}$ This new occupied feature is accordingly assigned to the HOMO of DAE1-o molecules. Considering the analyzed volume of the film (UPS surface sensitivity is $\sim 3 \mathrm{~nm}$ ), one can conclude that a switching efficiency for (at least near-surface) DAE1-c molecules of almost $100 \%$ could be achieved. Moreover, the photoisomerisation process resulted in a SECO shift by $0.15 \mathrm{eV}$ towards higher work function. A similar work function change was also observed for DAE1 on ITO substrates, ${ }^{[10]}$ but the shift was in the opposite direction. We attribute the WF change after photo-switching to an influence of the substrate WF and also the DAE1 molecular dipole moment (is discussed in detail further below). Subsequently, the illumination of the sample with UV light resulted in a ringclosure reaction, as indicated by the re-appearing DAE1-c HOMO feature with its onset close to the original position at $\sim 0.62 \mathrm{eV}$ BE. Also the WF shifted back to its original 
position $(4.5 \mathrm{eV})$ when the photostationary state was established after $120 \mathrm{~s}$ UV light irradiation. However, it is noteworthy that the spectrum in the valance region is still composed of both DAE1-o and DAE1-c contributions, according to a deconvolution of measured UPS spectrum. The fraction of 45\% DAE1-o and 55\% DAE1-c molecules (see fitting results in inset of Fig 2a) suggests that the back transfer (from DAE1-o to DAE1-c) was not completely reversible. This can be attributed to a manifold of causes, such as steric hindrance, ${ }^{[17,22-23]}$ radiation (UV, VUV) induced photoquenching, ${ }^{[24]}$ which prohibit such photoswitching of diarylethene molecules during light irradiation. Fully analogous photochromic switching was also observed for an ultrathin DAE1-c layer with only of $2 \mathrm{~nm}$ thickness (Fig. S2a); the thickness was estimated by considering both XPS spectra and AFM images (Fig. S2b and c). These results prove that the switchable properties of DAE1 molecules pertain in the bulk, at the bulk surface to vacuum, and also in immediate proximity to the metallic Au substrate.

Fig $2 \mathrm{~b}$ shows that in situ illumination of a film of initially pure DAE1-o using UV light as well gave rise to the attenuation of HOMO features of DAE1-o and the emergence of low BE-states (at $\sim 0.7 \mathrm{eV}$ ). These states are accordingly assigned to the HOMO of DAE1-c molecules. As the WF stayed the same (within $0.1 \mathrm{eV}$ ) the IE values of the two isomers vary substantially (as was the case above), between $5.9 \mathrm{eV}$ for the open and $5.1 \mathrm{eV}$ for the closed form. As was the case for the ultrathin DAE1-c above, the switching process for the ultrathin DAE1-o layer (2 nm, indicated in XPS in Fig. S3b) on $\mathrm{Au}$ is operative. Note that the position of $\mathrm{HOMO}$ onset, which is located at $1.29 \mathrm{eV} \mathrm{BE}$ in the valence region for the ultrathin film, is closer to the Fermi level compared to the HOMO position $(1.40 \mathrm{eV})$ of the thicker $(10 \mathrm{~nm})$ film. This is a typical observation as the 
photohole screening farther from the metal substrate is less efficient, which is reflected in the higher BE for larger film thicknesses.

A summary of the light induced energy level scenarios for DAE1 molecules on Au (thin and thick film) is depicted in Fig. 2c. Notably, the deposition of ultrathin DAE1-c and DAE1-o films reduced the WF of the metal surface $(4.70 \mathrm{eV})$ by $0.22 \mathrm{eV}$ and $0.12 \mathrm{eV}$, respectively, which is ascribed to the "push-back" or "cushion effect" ${ }^{[25,26]}$ Fro thicker films, no further changes in work function are observed, as a monolayer is sufficient to settle the push-back effect. By comparing the ELA between the Au/DAE1-o and Au/DAE1-c interface, one finds that the position of the HOMO onset of DAE1-c molecules $(0.53 \mathrm{eV} \mathrm{BE})$ lies much closer to the Fermi level of the Au substrate, than that of DAE1-c $(1.29 \mathrm{eV})$. The corresponding charge injection barriers, i.e., $\Delta_{\mathrm{e}}$, and $\Delta_{\mathrm{h}}$, at the Au/DAE1 interfaces can thus be efficiently controlled in a dynamic manner by illumination with light of appropriate energy (UV and visible).

To study the ELA at the organic semiconductor/DAE1 interfaces, i.e., P3HT/DAE1 and N2200/DAE1, DAE1 molecules were sequentially deposited on top of the polymer layers. After each step of deposition, samples were measured with UPS, corresponding spectra shown in Fig. 3. While we cannot rule out intermixing of polymers and DAE1 on a molecular level, the complete attenuation of polymer features in photoemission spectra at only 2-3 nm DAE1 coverage indicates rather sharp interfaces. Fig. 3a displays the thickness dependent UPS spectra of DAE1-o deposited on P3HT. The valence band onset of $\mathrm{P} 3 \mathrm{HT}$ is at $0.25 \mathrm{eV} \mathrm{BE}$ (Fermi level pinned due to its low IE of $4.60 \mathrm{eV}$ compared to the high work function of the PEDOT:PSS substrate $(5.0 \mathrm{eV})^{[27]]}$. The deposition of up to $2 \mathrm{~nm}$ DAE1-o on top of P3HT resulted in an attenuation of P3HT features, and the 
emergence of features centered at $2 \mathrm{eV}$ and $3.8 \mathrm{eV} \mathrm{BE}$, which are attributed to the valence levels of DAE1-o. Notably, the valence band onset of P3HT remained fixed at $0.25 \mathrm{eV} \mathrm{BE}$ and the WF remained constant, indicating weak interaction at the P3HT/DAE1-o interface. Increasing the coverage to $5 \mathrm{~nm}$ allowed the observation of a clearly discernible DAE1-o HOMO emission, with its onset at $1.51 \mathrm{eV} \mathrm{BE}$, with yet constant WF. Given that the IE of DAE1-o, deduced from UPS above, is $\sim 5.9 \mathrm{eV}$, its electron affinity, determined by considering the transport gap (see Fig. S6, also measured for DAE1-c), is $\sim 2.05 \mathrm{eV}$. Prior to contact with P3HT, the Fermi level (P3HT work function: $4.35 \mathrm{eV}$ ) lies well within the band gap of DAE1-o, thus leading to the observed vacuum level alignment. Subsequently, the P3HT/DAE1-o sample was irradiated using UV light for $240 \mathrm{~s}$, and DAE1-c molecules were thus formed with the HOMO level onset at $0.8 \mathrm{eV}$ BE. By fitting the spectrum we are able to calculate the switching efficiency, which is the same as on Au substrate (Fig. S5a). With the exception of small deviations in terms of absolute HOMO-onset values $(<0.15 \mathrm{eV})$, the observations for the reversed situation, i.e., pristine DAE1-c deposited on P3HT and then switched by visible light were fully analogous (Fig. S4a).

In contrast, the deposition of DAE1-o on N2200 gave rise to a small shift of the sample work function (Fig. 3b) towards lower values, indicating the formation of an interface dipole at the N2200/DAE1-o interface. However, no valence spectra shifts were observed with increasing deposition thickness. When the deposition thickness reached $5 \mathrm{~nm}$, the obtained WF and HOMO onset position of DAE1-o were $4.55 \mathrm{eV}$ and $1.43 \mathrm{eV}$, respectively. After UV light irradiation, DAE1-c conformers on N2200 were formed with a switching efficiency of 50\% (Fig. S5b), and not only did the HOMO onset change from 
$1.43 \mathrm{eV}$ to $0.73 \mathrm{eV}$ (as expected), but also the WF decreased by $0.15 \mathrm{eV}$ to $4.4 \mathrm{eV}$ [in line with pristine DAE1-c on N2200 (Fig. S4b)]. Since the HOMO level of DEA1 is quite far from $\mathrm{E}_{\mathrm{F}}$ in both conformations, it is not very likely that Fermi level pining occurs (yet, we cannot formally rule it out), we estimate whether changes of the molecular dipole moment due to the photochromic ring-opening/closure reaction could give rise to the observed WF change. ${ }^{[10]}$ We use the Helmholtz equation:

$$
\Delta \Phi=\frac{e n \Delta \mu}{\varepsilon_{0} \varepsilon_{r}}
$$

where $e$ is the elementary charge, $n$ is the surface dipole density $\left(1\right.$ molecule $\left./ \mathrm{nm}^{2}\right), \Delta \mu$ is the change of the dipole moment perpendicular to the surface (1.3 Debye per DAE1 molecule $\left.{ }^{[10]}\right), \varepsilon_{0}$ is the vacuum permittivity, and $\varepsilon_{r}$ is the relative dielectric constant. Assuming that all molecules are oriented with their dipole moments perpendicular to the surface, the calculated work function change $(\Delta \Phi)$ is $0.3 \mathrm{eV}$, only $0.1 \mathrm{eV}$ larger than the experimentally observed value. This suggests that indeed a change of the molecular dipole can explain the observed WF change, however, it is unlikely that all the molecules have the same orientation, despite a preferential orientation that may persist.

To discuss the functionality of the two organic semiconductor/DAE-1 interfaces, we refer to Fig. 4, where the results obtained by UPS and IPES are summarized. In Fig. 4a we observe that vacuum level alignment prevails for both open and closed DAE1 in P3HT. From the estimation of molecular dipole moments above we conclude that no preferential orientation of the switches occurs. When DAE1-o is in contact with P3HT there is a huge energy difference $(1.26 \mathrm{eV})$ between the polymer valence band edge and the molecular HOMO level such that hole transfer from P3HT to DAE1-o is largely 
suppressed. When the molecule is switched into the closed form, the HOMO level moves much closer to the valence band and hole transfer becomes improved, yet the energy offset $(0.55 \mathrm{eV})$ is still substantial. Conversely, the conduction band edge of P3HT (2.35 eV above $\left.\mathrm{E}_{\mathrm{F}}\right)$ and the LUMO level of DAE1-o (2.34 eV above $\left.\mathrm{E}_{\mathrm{F}}\right)$ are in resonance, enabling good electron transfer; when switched to the closed form the LUMO level moves to $1.46 \mathrm{eV}$ above $\mathrm{E}_{\mathrm{F}}$ and electron transfer from the molecule to the polymer becomes inhibited. The N2200/DAE1-o interface is a type-I heterojunction with substantial energy offsets (several $100 \mathrm{meV}$ ) between the hole and electron transport levels (see Fig. 4b), implying very inefficient charge transfer from the polymer to the molecule. However, upon switching DAE1 into its closed form, the respective transport levels become almost resonant in energy, i.e., the energy offset between conduction band edge and LUMO level is $0.6 \mathrm{eV}$ and that between valence band edge and HOMO level is $0.07 \mathrm{eV}$, enabling facile electron and hole transfer across the junction. In all energy level scenarios depicted in Fig. 4, ground state charge transfer between DAE1 and the respective polymer does not occur. Consequently, the energy level alignment between the organic semiconductors and the DAE1 photochromic switch can be altered over a very wide range by an appropriate light stimulus.

\section{Conclusion}

We performed direct and inverse photoemission studies to reveal how the interfacial level alignment between thin films consisting of the photochromic molecular switch DAE1 and Au electrodes as well as two polymeric semiconductors (p-type P3HT and n-type N2200) 
can be modulated by light. Switching was achieved in situ by the irradiation with UV and visible light. First, we demonstrated that switching between open and closed forms of DAE1 on $\mathrm{Au}$ is operative, with high switching yields for both bulk as well as approximately monolayer thin molecular films. While switching-induced work function changes are negligible, the HOMO level onset position of DAE1 varies by $0.8 \mathrm{eV}$ with respect to the Au Fermi level between the two DAE isomers. Quantitative and fully reversible switching was also achieved for DAE1 on the two polymers. The charge transfer functionality of these junctions could be substantially modified by optical switching of DAE1, ranging from charge blocking to almost resonant energy levels for both, electrons and holes (in the case of N2200). Our findings provide a solid basis for the rational use of photochromic molecules for manipulating energy levels at electrode and organic semiconductor interfaces, and will aid the development of improved photoswitchable (opto-) electronic devices.

\section{Acknowledgements}

This work was financially supported by the EC through the Marie Curie project ITN iSwitch (GA no. 642196) and the DFG (Sfbs 658 and 951). 


\section{References}

[1] K. J. Baeg, M. Binda, D. Natali, M. Caironi \& Y. Y. Noh, Adv. Mater. 2013, $25,4267-4295$.

[2] S. Kawata \& Y. Kawata, Chem. Rev. 2000, 100, 1777 - 1788.

[3] R. C. Shallcross, P. Zacharias, A. Köhnen, P. O. Körner, E. Maibach, and K. Meerholz, Adv. Mater. 2013, 25, 469-476.

[4] E. Orgiu and P. Samorì, Adv. Mater. 2014, 26, 1827-1845.

[5] M. M. Russew and S. Hecht, Adv. Mater. 2010, 22, 3348-3360.

[6] M. Herder, B. M. Schmidt, L. Grubert, M. Pätzel, J. Schwarz, and S. Hecht, J. Am. Chem. Soc. 2015, 137, 2738-2747.

[7] S. V. Snegir, A. A. Marchenko, P. Yu, F. Maurel, O. L. Kapitanchuk, S. Mazerat, M. Lepeltier, A. Léaustic, and E. Lacaze, J. Phys. Chem. Lett. 2011, 2, 2433-2436.

[8] C. Weber, T. Liebig, M. Gensler, L. Pithan, S. Bommel, D. Bléger, J. P. Rabe, S. Hecht, and S. Kowarik, Macromolecules, 2015, 48, 1531-1537.

[9] M. J. Comstock, N. Levy, A. Kirakosian, J. Cho, F. Lauterwasser, J. H. Harvey, D. A. Strubbe, J. M. J. Fréchet, D. Trauner, S. G. Louie, and M. F. Crommie, Phys. Rev. Lett. 2007, 99, 038301.

[10] J. Frisch, M. Herder, P. Herrmann, G. Heimel, S. Hecht, N. Koch, Appl Phys A 2013, $113,1-4$ 
[11] S. Tanaka, M. Toba, T. Nakashima, T. Kawai, and K. Yoshino, Jpn. J. Appl. Phys. 2008, 47, 1215-1218.

[12] M. E. Gemayel, K. Börjesson, M. Herder, D. T. Duong, J. A. Hutchison, C. Ruzié, G. Schweicher, A. Salleo, Y. Geerts, S. Hecht, E. Orgiu, P. Samorì, Nat. Commun.2015, $6,6330$.

[13] E. Orgiu, N. Crivillers, M. Herder, L. Grubert, M. Pätzel, J. Frisch, E. Pavlica, D. T. Duong, G. Bratina, A. Salleo, N. Koch, S. Hecht, P. Samorì, Nat. Chem. 2012, 4, 675.

[14] N. Crivillers, E. Orgiu, F. Reinders, M. Mayor, and P. Samorì, Adv. Mater. 2011, 23, $1447-1452$.

[15] N. Crivillers, S. Osella, C. V. Dyck, G. M. Lazzerini, D. Cornil, A. Liscio, F. D. Stasio, S. Mian, O. Fenwick, F. Reinders, M. Neuburger, E. Treossi, M. Mayor, V. Palermo, F. Cacialli, J. Cornil, and P. Samorì, Adv. Mater. 2013, 25, 432-436.

[16] R. Hayakawa, M. Petit, K. Higashiguchi, K. Matsuda, T. Chikyow, Y. Wakayama, Org. Electron. 2015, 21, 149-154.

[17] S. Fredrich, R. Göstl, M. Herder, L. Grubert, and Stefan Hecht, Angew. Chem. Int. Ed. 2015, 54, 1-6.

[18] R. Hayakawa, K. Higashiguchi, K. Matsuda, T. Chikyow, Y. Wakayama, ACS Appl. Mater. Interfaces 2013, 5, 3625-3630.

[19] H. Wang, P. Amsalem, G. Heimel, I. Salzmann, N. Koch and M. Oehzelt, $A d v$. Mater. 2014, 26, 925. 
[20] H. Ishii, K. Sugiyama, E. Ito, K. Seki, Adv. Mater. 1999, 11, 605.

[21] S. Braun, W.R. Salaneck, M. Fahlman, Adv. Mater. 2009, 21, 1450.

[22] X. Li, W. Li, H. Ågren, H. Tian, W. Zhu, Dyes Pigments 2016, 125, 348-355.

[23] W. Li, Y. Cai, X. Li, H. Ågren, H. Tian and W. Zhu, J. Mater. Chem. C, 2015, 3, 8665-8674.

[24] Z. Li, L. Liao, W. Sun, C. Xu, C. Zhang, C. Fang, and C. Yan, J. Mater. Chem. C, 2008, 112, 5190-5196.

[25] S. Winkler, J. Frisch, R. Schlesinger, M. Oehzelt, R. Rieger, J. Räder, J. P. Rabe, K. Müllen, and N. Koch, J. Phys. Chem. C 2013, 117, 22285.

[26] G. Witte, S. Lukas, P. S. Bagus, and C. Wöll, Appl. Phys. Lett. 2005, 87, 263502.

[27] J. Frisch, A. Vollmer, J. P. Rabe, N. Koch, Org. Electron. 2011, 12, 916-922.

[28] J. Frisch, A. Vollmer, and N. Koch, J. Appl. Phys. 2012, 112, 033712.

[29] C. Deibel, D. Mack, J. Gorenflot, A. Schöll, S. Krause, F. Reinert, D. Rauh and V. Dyakonov, Phys. Rev. B 2010, 81, 085202.

[30] S. Fabiano, H. Yoshida, Z. Chen, A. Facchetti, and M. A. Loi, ACS Appl. Mater. Interfaces 2013, 5, 4417-4422. 
(a)
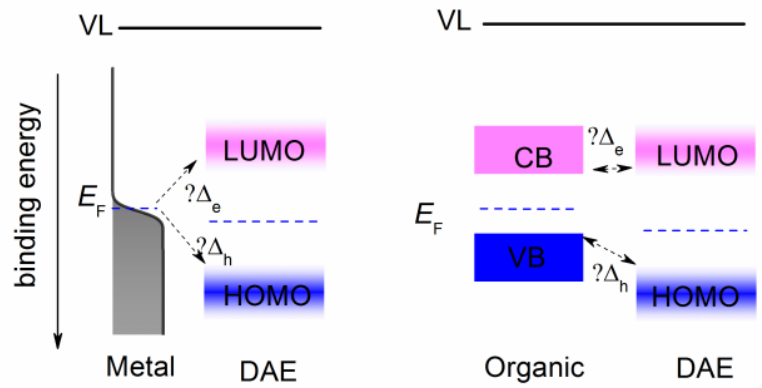

(b)

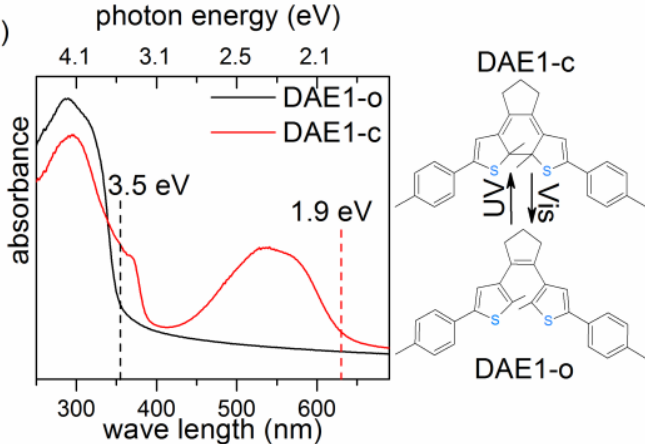

(c)

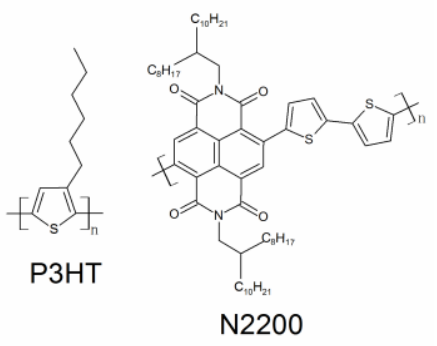

Figure 1. (a) Generic energy level diagram at metal/DAE1 and organic/DAE1 interfaces before contact, with the a priori unknown energy barriers ( $\Delta_{\mathrm{e}}$ for electrons, $\Delta_{\mathrm{h}}$ for holes). (b) Left: UV-vis absorption spectra of DAE1-o and DAE1-c thin films (10 nm on quartz glass), the optical gaps are indicated from the absorption edges (dashed lines). Right: Chemical structure of DAE1 in the two conformations. (c) Chemical structure of P3HT and N2200. 

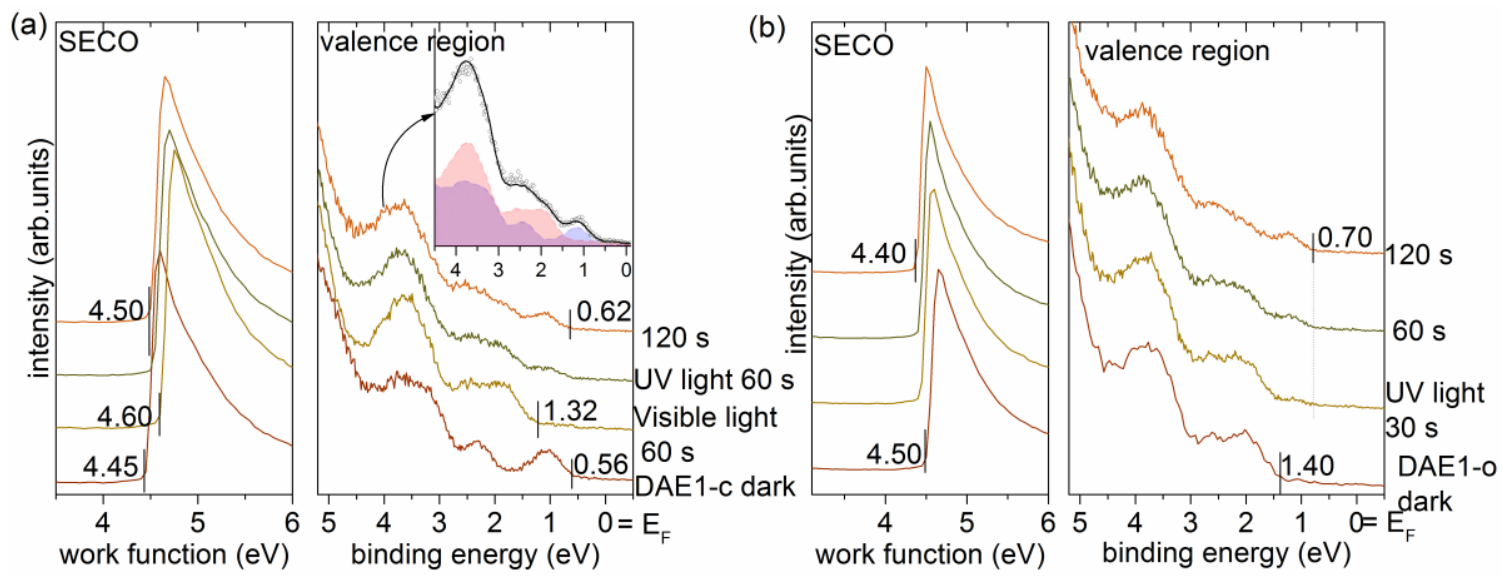

(c)



Au

$2 \mathrm{~nm} \underset{\text { DAE1-c }}{10 \mathrm{~nm}}$

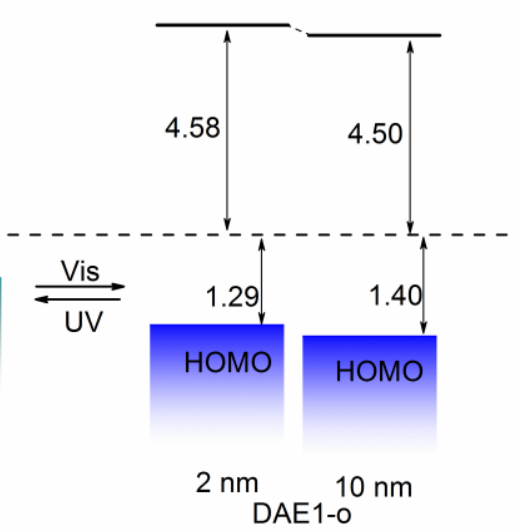

Figure 2. Electronic structure of $10 \mathrm{~nm}$ thick DAE1 films on Au substrates upon switching with light. (a) UPS spectra (valence and SECO regions) for initially DAE1-c. Inset: deconvolution of sample spectrum (open circles) after $120 \mathrm{~s}$ ultraviolet light irradiation with DAE1-c and DAE1-o contributions (sum given by the black line). (b) UPS spectra of initially DAE1-o. (c) Switching-induced energy level alignment diagram of DAE1-c and DA1-o films on Au, as determined from UPS. 

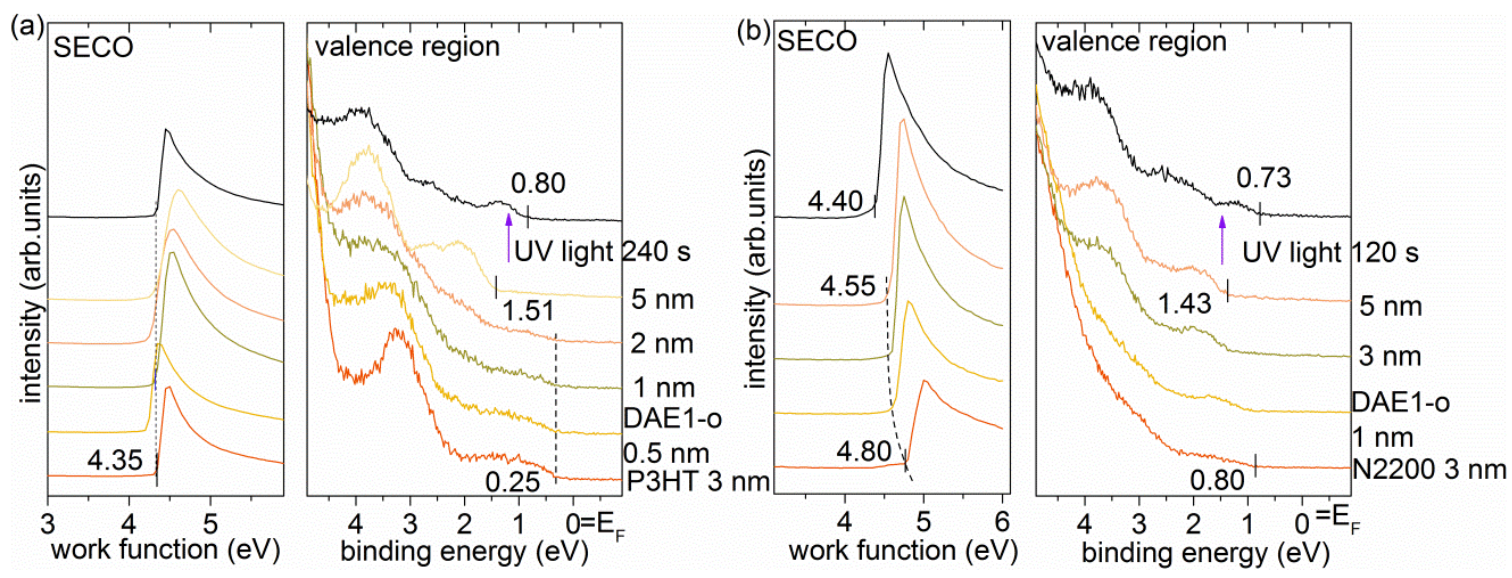

Figure 3. UPS spectra (valence and SECO regions) of DAE1-o films with incremental thickness on (a) P3HT and (b) N2200, and light switching induced changes. 
(a)
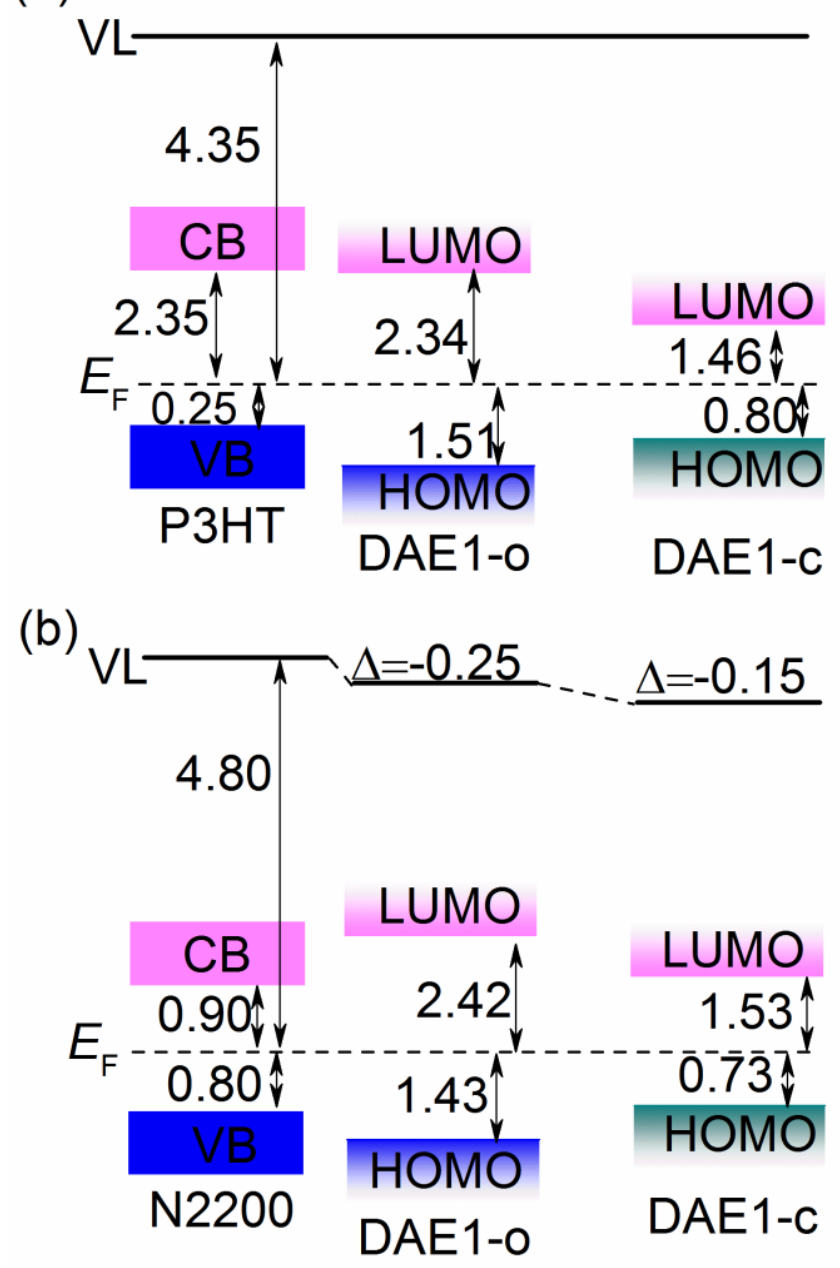

Figure 4. Schematic energy level diagrams of (a) P3HT/DAE1 and (b) N2200/DAE1 interfaces. The energetic positions of $\mathrm{VL}$ and $\mathrm{HOMO}(\mathrm{VB})$ onset relative to $E_{\mathrm{F}}$ were determined by UPS measurements, while CB onsets of P3HT and N2200 were calculated by considering the transport gaps. ${ }^{[29,30]}$ The LUMO onsets of DAE1-o and DAE1-c films, were calculated by using their charge transport gaps, which were measured by UPS and IPES (Fig. S5). 


\section{Text and figure for table of contents}

The photoisomerization was achieved by in situ irradiation of ultraviolet $(\lambda=365 \mathrm{~nm})$ and visible light $(\lambda>450 \mathrm{~nm})$. Efficient and reversible switching between the open and closed isomers of DAE1 was evidenced at all interfaces, with profound impact on the energy level alignment.



